\title{
EIGHT-DIMENSIONAL REAL ABSOLUTE-VALUED ALGEBRAS WITH LEFT UNIT WHOSE AUTOMORPHISM GROUP IS TRIVIAL
}

\author{
A. ROCHDI
}

Received 5 May 2002

\begin{abstract}
We classify, by means of the orthogonal group $\mathrm{O}_{7}(\mathbb{R})$, all eight-dimensional real absolute-valued algebras with left unit, and we solve the isomorphism problem. We give an example of those algebras which contain no four-dimensional subalgebras and characterise with the use of the automorphism group those algebras which contain one.
\end{abstract}

2000 Mathematics Subject Classification: 17A35.

1. Introduction. One of the fundamental results about finite-dimensional real division algebras is due to Kervaire [7] and Bott and Milnor [3], and states that the $n$-dimensional real vector space $\mathbb{R}^{n}$ possesses a bilinear product without zero divisors only in the case where the dimension $n=1,2,4$, or 8 . All eightdimensional real division algebras that occur in the literature contain a fourdimensional subalgebra (see [1, 2, 4, 5, 6]). However, it is still an open problem whether a four-dimensional subalgebra always exists in an eight-dimensional real division algebra, even for quadratic algebras [4]. In [9], Ramírez Álvarez gave an example of a four-dimensional absolute-valued real algebra containing no two-dimensional subalgebras. On the other hand, any four-dimensional absolute-valued real algebra with left unit contains a two-dimensional subalgebra. Therefore, a natural question to ask is whether an eight-dimensional real absolute-valued algebra with left unit contains a four-dimensional subalgebra. In this note, we give a negative answer and we characterise the eightdimensional absolute-valued real algebras with left unit containing a fourdimensional subalgebra in terms of the automorphism group.

2. Notation and preliminary results. For simplicity, we only consider vector spaces over the field $\mathbb{R}$ of real numbers.

DEFinition 2.1. Let $A$ be an algebra; $A$ is not assumed to be associative or unital.

(1) An element $x \in A$ is called invertible if the linear operators

$$
L_{x}: y \longmapsto x y, \quad R_{x}: y \longmapsto y x
$$


are invertible in the associative unital algebra $\operatorname{End}(A)$. The algebra $A$ is called a division algebra if all nonzero elements in $A$ are invertible.

(2) A unital algebra $A$ is called a quadratic algebra if $\left\{1, x, x^{2}\right\}$ is linearly dependent for all $x \in A$. If $(\cdot / \cdot)$ is a symmetric bilinear form over $A$, then a linear operator $f$ on $A$ is called an isometry with respect to $(\cdot / \cdot)$ if $(f(x) / f(y))=$ $(x / y)$ for all $x, y \in A$. If, moreover, $(x y / z)=(x / y z)$, for all $x, y, z \in A$, then $(\cdot / \cdot)$ is called a trace form over $A$.

(3) The algebra $A$ is termed normed (resp., absolute-valued) if it is endowed with a space norm $\|\cdot\|$ such that $\|x y\| \leq\|x\|\|y\|($ resp., $\|x y\|=\|x\|\|y\|)$ for all $x, y \in A$. A finite-dimensional absolute-valued algebra is obviously a division algebra and has a subjacent Euclidean structure (see [11]).

(4) An automorphism $f \in \operatorname{Aut}(A)$ is called a reflexion of $A$ if $f \neq I_{A}$ and $f^{2}=I_{A}$.

Write $\operatorname{Aut}(\mathbb{O})=G_{2}$. We denote by $S(E)$ and vect $\left\{x_{1}, \ldots, x_{n}\right\}$, respectively, the unit sphere of a normed space $E$ and the vector subspace spanned by $x_{1}, \ldots, x_{n} \in E$.

It is known that a quadratic algebra $A$ is obtained from an anticommutative algebra $(V, \wedge)$ and a bilinear form $(\cdot, \cdot)$ over $V$ as follows: $A=\mathbb{R} \oplus V$ as a vector space, with product

$$
(\alpha+x)(\beta+y)=(\alpha \beta+(x, y))+(\alpha y+\beta x+x \wedge y)
$$

We have a bilinear form associated to A, namely,

$$
A \times A \longrightarrow \mathbb{R}, \quad(\alpha+x, \beta+y) \longmapsto \alpha \beta+(x, y),
$$

$(V, \wedge)$ is called the anticommutative algebra associated to $A$. The elements of $V$ are called vectors, while the elements of $\mathbb{R}$ are called scalars. We write $A=(V,(\cdot, \cdot), \wedge)$ (see [8]).

We will write $(W,(\cdot / \cdot), \times)$ for the (quadratic) Cayley-Dickson octonions algebra (1) with its trace form $(\cdot / \cdot)$ and the anticommutative algebra $(W, \times)$. For $u \neq 0 \in W, W(u)$ will be the orthogonal subspace of $\mathbb{R} \cdot u$ in $W$. It is well known that $\mathbb{O}$ is an alternative algebra, that is, it satisfies the identities $x^{2} y=x(x y)$ and $y x^{2}=(y x) x$.

REMARK 2.2. Let $A$ be an eight-dimensional absolute-valued algebra with left unit $e$, and $f$ is an isometry of the Euclidian space $A$ such that $f(e)=e$. Let $A_{f}$ be equal to $A$ as a vector space, with a new product given by the formula $x * y=f(x) y$, for all $x, y \in A$. Then $A_{f}$ is also an absolute-valued algebra with left unit $e$. It is clear that an $f$-invariant subalgebra of $A$ is a subalgebra of $A_{f}$. In particular, if we consider the isometry $R_{e}^{-1}$, then we obtain an absolute-valued algebra $A_{R_{e}^{-1}}$ with unit $e$, which is isomorphic to $\mathbb{O}$ (see [12]). 
3. Isometries of $\mathbb{O}$ with no invariant four-dimensional subalgebras. Let $\varphi$ be an isometry of the Euclidian space $\mathbb{O}=\mathbb{R} \oplus W$, fixing the element 1 . Then there exists an orthonormal basis $\mathscr{B}=\left\{1, x_{1}, \ldots, x_{7}\right\}$ of $\mathbb{O}$ such that $x_{1}$ is an eigenvector of $\varphi$ and $W_{k}=\operatorname{vect}\left\{x_{2 k}, x_{2 k+1}\right\}$ is a $\varphi$-invariant subspace of $\mathbb{O}$, for $k=1,2,3$. If $B$ is a four-dimensional $\varphi$-invariant subspace of $\mathbb{O}$ containing 1 , then the basis $\mathscr{B}$ can be chosen as an extension of an orthonormal basis $\{1, u, y, z\}$ of $B$, with $u \in W$ an eigenvector of $\varphi$, and $E=\operatorname{vect}\{y, z\}$ is a $\varphi$-invariant subspace of $B$. Thus, $B$ can be written as a direct orthogonal $\varphi$-invariant sum $\mathbb{R} \oplus \mathbb{R} \cdot u \oplus E$.

In the following important example, we use the notation introduced above.

EXAMPLE 3.1. If $\varphi$ fixes $x_{1}$ and its restriction to every $W_{k}$ is the rotation with angle $k \pi / 4$, then vect $\left\{1, x_{1}\right\}$ is the eigenspace $E_{1}(\varphi)$ of $\varphi$ associated to the eigenvalue 1 . The characteristic polynomial $P_{\varphi}(X)$ of $\varphi$ is then

$$
\begin{gathered}
(X-1)^{2}\left(X^{2}-2 X \cos \left(\frac{\pi}{4}\right)+1\right)\left(X^{2}-2 X \cos \left(\frac{2 \pi}{4}\right)+1\right)\left(X^{2}-2 X \cos \left(\frac{3 \pi}{4}\right)+1\right) \\
=\prod_{0 \leq k \leq 3} P_{k}(X)
\end{gathered}
$$

with

$$
P_{k}(X)=X^{2}-2 X \cos \left(\frac{k \pi}{4}\right)+1
$$

The characteristic polynomial $P_{\varphi_{/ / B}}(X)$ of the restriction of $\varphi$ to $B$ is a polynomial of degree 4 , a multiple of $X-1$, and a divisor of $P_{\varphi}(X)$. Actually, $P_{\varphi_{/ / B}}(X)=(X-1)^{2} P_{k}(X)$ for $k \in\{1,2,3\}$, and this "forces" $B$ to be of the form $E_{1}(\varphi) \oplus W_{k}$ for a certain $k \in\{1,2,3\}$. In particular, if $\mathscr{B}$ is obtained from the canonical basis $\left\{1, e_{1}, \ldots, e_{7}\right\}$ of $\mathbb{O}$ by taking

$$
\begin{gathered}
x_{1}=e_{5}, \quad x_{2}=\frac{e_{1}+e_{2}}{\sqrt{2}}, \quad x_{3}=\frac{e_{1}-e_{2}}{\sqrt{2}}, \quad x_{4}=\frac{e_{3}+e_{4}}{\sqrt{2}}, \\
x_{5}=\frac{e_{3}-e_{4}}{\sqrt{2}}, \quad x_{6}=\frac{e_{6}+e_{7}}{\sqrt{2}}, \quad x_{7}=\frac{e_{6}-e_{7}}{\sqrt{2}},
\end{gathered}
$$

then for each $i \neq j$ and $l, x_{i} \times x_{j}$ and $x_{l}$ are not colinear. This shows that $E_{1}(\varphi) \oplus W_{k}$ is not a subalgebra of $\mathbb{O}$, for $k=1,2,3$. It follows that $\mathbb{O}$ has no four-dimensional $\varphi$-invariant subalgebras.

4. Eight-dimensional real absolute-valued algebras with left unit. First recall the following result from [11].

LEMMA 4.1. Every homomorphism from a normed complete algebra into an absolute-valued algebra is contractive. In particular, every isomorphism of absolute-valued algebras is an isometry.

As a consequence we have the following lemma. 
LEMMA 4.2. Let $\psi: A \rightarrow B$ be an isomorphism of absolute-valued $\mathbb{R}$-algebras and $f: A \rightarrow A$ an isometry. Then $\psi \circ f \circ \psi^{-1}: B \rightarrow B$ is an isometry and $\psi: A_{f} \rightarrow$ $B_{\psi \circ f \circ \psi^{-1}}$ is an isomorphism. In particular, $\psi: A_{f} \rightarrow \mathbb{O}$ is an isomorphism if and only if $\psi: A \rightarrow \mathbb{O}_{\psi \circ f^{-1} \circ \psi^{-1}}$ is an isomorphism.

Proof. The first statement is a consequence of Lemma 4.1. For $x, y \in A$, we have

$$
\psi(f(x) y)=\psi(f(x)) \psi(y)=\left(\psi \circ f \circ \psi^{-1}\right)(\psi(x)) \psi(y)
$$

hence $\psi: A_{f} \rightarrow B_{\psi \circ f \circ \psi^{-1}}$ is an isomorphism.

THEOREM 4.3. Every eight-dimensional absolute-valued left unital algebra is isomorphic to $\mathbb{O}_{f}$ where $f$ is an isometry of the Euclidian space $\mathbb{O}$ which fixes 1. Moreover, the following two properties are equivalent:

(1) $\mathbb{O}_{f}$ and $\mathbb{O}_{g}$ are isomorphic $(f, g$ being two isometries of $\mathbb{O}$ fixing 1 );

(2) there exists $\psi \in G_{2}$ such that $g=\psi \circ f \circ \psi^{-1}$, that is, $f$ and $g$ are in the same orbit of conjugations by isometries of $(\mathbb{O}$ fixing 1 .

Proof. The first statement is a consequence of a Remark 2.2 and Lemma 4.2. The second statement can be proved as follows: $\psi: \mathbb{O}_{f} \rightarrow \mathbb{O}_{g}$ is an isomorphism if and only if $\psi: \mathbb{O} \rightarrow\left(\mathbb{O}_{g}\right)_{\psi \circ f^{-1} \circ \psi^{-1}}=\mathbb{O}_{\psi \circ f^{-1} \circ \psi^{-1} \circ g}$ is an isomorphism. This is equivalent to

$$
\psi \circ f^{-1} \circ \psi^{-1} \circ g=I_{\mathbb{O}}, \quad \psi \in G_{2}
$$

5. Subalgebras and automorphisms of $\mathbb{O}_{\varphi}$. The following preliminary result allows us to characterise the subalgebras of $\mathbb{O}_{\varphi}$.

LEMMA 5.1. If $A$ is an algebra with left unit and without zero divisors, then every nontrivial finite-dimensional subalgebra of A contains the left unit element of $A$.

Proof. Such a subalgebra $B$ is a division algebra and for every $x \neq 0 \in B$, there exists $y \in B$ such that $y x=x$. On the other hand, if $e$ is the left unit of $A$, then $e x=x$. Then the absence of zero divisors in $A$ shows that $y=e \in B$.

What are the subalgebras of $\mathbb{O}_{\varphi}$ ?

Proposition 5.2. Let $\varphi$ be an isometry of the Euclidian space $(\mathbb{O}$ that fixes 1 and $B$ is a subspace of $\mathbb{O}$. Then the following two properties are equivalent:

(1) $B$ is a subalgebra of $\mathbb{O}_{\varphi}$;

(2) $B$ is a $\varphi$-invariant subalgebra of $\mathbb{O}$.

Proof. $(1) \Rightarrow(2)$. The subalgebra $B$ contains the left unit element 1 of $\mathbb{O}_{\varphi}$ and is $\varphi$-invariant. Indeed, 


$$
1 \in B, \quad \forall x \in B: \varphi(x)=\underbrace{\varphi(x) 1}_{\text {product in } \mathbb{O}}=\overbrace{x * 1}^{\text {product in } \mathbb{O}_{\varphi}} \in B .
$$

(2) $\Rightarrow(1)$. See Remark 2.2.

REMARK 5.3. (1) The algebra $\mathbb{O}_{\varphi}$ has a two-dimensional subalgebra because $\varphi$ has an eigenvector $x \in W$ and the subalgebra vect $\{1, x\}$ of $\mathbb{O}$ is $\varphi$-invariant. This argument shows that $\mathbb{H}_{\varphi}$ has a two-dimensional subalgebra.

(2) Let $\varphi$ be the isometry considered in Example 3.1. Then $\mathbb{O}_{\varphi}$ has no fourdimensional subalgebras.

The following elementary result is useful for characterising the automorphisms of the algebra $\mathbb{O}_{\varphi}$.

LEMMA 5.4. Let $A$ be an algebra with left unit e and without zero divisors. If $f \in \operatorname{Aut}(A)$, then $f(e)=e$.

Proof. We have $(f(e)-e) f(e)=0$.

What are the automorphisms of the algebra $\mathbb{O}_{\varphi}$ ?

Proposition 5.5. If $\varphi$ is an isometry of the Euclidian space $(\mathbb{O}$ that fixes 1 , then $f \in \operatorname{Aut}\left(\mathbb{O}_{\varphi}\right)$ if and only if $f \in G_{2}$ and $f$ commutes with $\varphi$.

Proof. For all $x, y \in \mathbb{O}$, we have that $f(\varphi(x) y)=\varphi(f(x)) f(y)$, hence $f(\varphi(x))=f(\varphi(x) 1)=\varphi(f(x)) f(1)=\varphi(f(x))$, and $f \circ \varphi=\varphi \circ f$ and $f \in$ $G_{2}$.

REMARK 5.6. If $f \in \operatorname{Aut}\left(\mathbb{O}_{\varphi}\right) \backslash\left\{I_{\mathbb{O}}\right\}$ is a reflexion, then $B=\operatorname{Ker}\left(f-I_{\mathbb{O}}\right)$ is a four-dimensional subalgebra of $\mathbb{O}_{\varphi}$.

6. The relation in $\mathbb{O}_{\varphi}$ between four-dimensional subalgebras and nontrivial automorphisms. We begin with the following useful preliminary result taken from [10].

LEMMA 6.1. Every four-dimensional subalgebra $B$ of $\mathbb{O}=(W,(\cdot / \cdot), \times)$ coincides with the square of its orthogonal $B^{\perp}$ and satisfies the equality $B B^{\perp}=B^{\perp} B=$ $B^{\perp}$.

Proof. Let $v \in S\left(B^{\perp}\right)$, then $B^{\perp}=v B$. Indeed, taking into account the trace property of $(\cdot / \cdot)$, we have for all $x, y \in B$ that $(v x / y)=(v / x y)=0$, hence $v B \subset B^{\perp}$, and we have equality because the dimensions of both spaces are equal. Using the middle Moufang identity, we compute that

$$
(v x)(v y)=-(v x)(y v)=v(x y) v=x y
$$

for all $x, y \in B$. Taking into account the anticommutativity of the product $\times$, we find that $B B^{\perp}=B^{\perp} B$. Finally, the trace property of $(\cdot / \cdot)$ shows that $B B^{\perp}$ is orthogonal to $B$, hence $B B^{\perp} \subset B^{\perp}$. 
Proposition 6.2. Let B be a $\varphi$-invariant four-dimensional subalgebra of $(\mathbb{O}$. Then the map

$$
f: \mathbb{O}=B \oplus B^{\perp} \longrightarrow \mathbb{O}, \quad f(a+b)=a-b,
$$

is a reflexion which commutes with $\varphi$.

Proof. Take $a, x \in B$ and $b, y \in B^{\perp}$. Using Lemma 6.1, we compute

$$
\begin{aligned}
f((a+b)(x+y)) & =f(a x+b y+a y+b x) \\
& =(a x+b y)-(a y+b x) \\
& =(a-b)(x-y) \\
& =f(a+b) f(x+y),
\end{aligned}
$$

$B^{\perp}$ is $\varphi$-invariant since $B$ is $\varphi$-invariant, and we have

$$
\begin{aligned}
(f \circ \varphi)(a+b) & =f(\varphi(a)+\varphi(b)) \\
& =\varphi(a)-\varphi(b) \\
& =\varphi(a-b) \\
& =(\varphi \circ f)(a+b) .
\end{aligned}
$$

THEOREM 6.3. If $\varphi$ is an isometry of the Euclidian space (1) which fixes 1 , then the following four properties are equivalent:

(1) $\mathbb{O}_{\varphi}$ contains a four-dimensional subalgebra;

(2) (1) contains a $\varphi$-invariant four-dimensional subalgebra;

(3) $\operatorname{Aut}\left(\mathbb{O}_{\varphi}\right)$ contains a reflexion;

(4) $\operatorname{Aut}\left(\mathbb{O}_{\varphi}\right)$ is not trivial.

Proof. The only thing that remains to be shown is that (4) implies (1). Let $g \in \operatorname{Aut}\left(\mathbb{O}_{\varphi}\right)-\left\{I_{\mathbb{O}}\right\}$. If $g$ is a reflexion, then the result follows from Remark 5.6. By assuming that $g$ is not a reflexion, we distinguish two cases.

CASE 1. The automorphism $g$ admits two linearly independent orthonormal eigenvectors $u, y \in W$. Then $g(u y)=g(u) g(y)=( \pm u)( \pm y)= \pm u y$ and vect $\{1, u, y, u y\}=\operatorname{Ker}\left(g^{2}-I_{\mathbb{O}}\right)$ is a $\varphi$-invariant four-dimensional subalgebra of $\mathbb{O}$.

CASE 2. The automorphism $g$ has only one eigenvector $u \in S(W)$ except the sign. Then $u$ is an eigenvector of $\varphi$ and $g$ and $\varphi$ induce isometries

$$
g_{u}, \varphi_{u}: W(u) \longrightarrow W(u) .
$$

Using the minimal polynomials $P(X)$ and $Q(X)$ of $g_{u}$ and $\varphi_{u}$, we will first show that $W(u)$ contains a two-dimensional $g$-invariant and $\varphi$-invariant subspace of $E$. The irreducible factors of $P(X)$ are polynomials of degree two with negative discriminant. However $Q(X)$ can have a factor of degree one, and then the 
existence of $E$ is assured by the fact that the eigenspaces of $\varphi_{u}$ are $f$-invariant, and their direct sum is of even dimension. So we can assume that $Q(X)$ is a product of polynomials of degree two with negative discriminant. Now, we have three different cases.

(a) $P(X)=X^{2}-\alpha X-\beta$ and $Q(X)=X^{2}-\lambda X-\mu$ are polynomials of degree two. Since $\alpha^{2}+4 \beta<0$ and $\lambda^{2}+4 \mu<0$, there exists $\omega \in \mathbb{R}^{*}$ such that $\alpha^{2}+4 \beta=$ $\omega^{2}\left(\lambda^{2}+4 \mu\right)$, and we have

$$
\begin{aligned}
\left(g_{u}-\frac{\alpha}{2} I_{W(u)}\right)^{2} & =\left(\frac{\alpha^{2}}{4}+\beta\right) I_{W(u)} \\
& =\omega^{2}\left(\frac{\lambda^{2}}{4}+\mu\right) I_{W(u)} \\
& =\omega^{2}\left(\varphi_{u}-\frac{\lambda}{2} I_{W(u)}\right)^{2} .
\end{aligned}
$$

Now $g_{u}$ and $\varphi_{u}$ commute, so

$$
\left(g_{u}-\frac{\alpha}{2} I_{W(u)}-\omega\left(\varphi_{u}-\frac{\lambda}{2} I_{W(u)}\right)\right) \circ\left(g_{u}-\frac{\alpha}{2} I_{W(u)}+\omega\left(\varphi_{u}-\frac{\lambda}{2}\right)\right) \equiv 0 .
$$

(i) If $g_{u}-(\alpha / 2) I_{W(u)}= \pm \omega\left(\varphi_{u}-(\lambda / 2) I_{W}(u)\right)$, then every $g$-invariant twodimensional subspace of $W(u)$ is $\varphi$-invariant, as well as its orthogonal.

(ii) If $g_{u}-(\alpha / 2) I_{W(u)} \neq \pm \omega\left(\varphi_{u}-(\lambda / 2) I_{W(u)}\right)$, then

$$
H=\operatorname{Ker}\left(g_{u}-\frac{\alpha}{2} I_{W(u)}-\omega\left(\varphi_{u}-\frac{\lambda}{2} I_{W(u)}\right)\right)
$$

and $H^{\perp}$ are $g_{u}$-invariant and $\varphi_{u}$-invariant proper subspaces of $W(u)$. One of them is necessarily two-dimensional and the other one is fourdimensional.

(b) If $\operatorname{deg}(P(X))>2$, then we consider an irreducible component $P_{1}(X)$ of $P(X)$. The kernel $\operatorname{Ker}\left(P_{1}\left(g_{u}\right)\right)$ and its orthogonal are then $g_{u}$-invariant and $\varphi_{u}$-invariant proper subspaces of $W(u)$.

(c) The case $\operatorname{deg}(Q(X))>2$ is similar to the case $\operatorname{deg}(P(X))>2$.

The subspace vect $\{1, u\} \oplus E$ is then a subalgebra of $\mathbb{O}$. Indeed, $E=\operatorname{vect}\{y, z\}$, with $y, z \in W(u)$ orthogonal, and there exist $a, b \in \mathbb{R}$ with $a^{2}+b^{2}=1$ such that the matrix of the restriction of $g$ to $E$ is

$$
\left(\begin{array}{cc}
a & -b \\
b & a
\end{array}\right) \text { or } \quad\left(\begin{array}{cc}
a & b \\
b & -a
\end{array}\right)
$$

Thus, $g(y z)=g(y) g(z)= \pm\left(a^{2}+b^{2}\right) y z= \pm y z$, and consequently $y z= \pm u$. Using alternativity and anticommutativity for vectors, we then obtain that $u y= \pm z$ and $u z= \pm y$. 


\section{REFERENCES}

[1] G. M. Benkart, D. J. Britten, and J. M. Osborn, Real flexible division algebras, Canad. J. Math. 34 (1982), no. 3, 550-588.

[2] G. M. Benkart and J. M. Osborn, An investigation of real division algebras using derivations, Pacific J. Math. 96 (1981), no. 2, 265-300.

[3] R. Bott and J. Milnor, On the parallelizability of the spheres, Bull. Amer. Math. Soc. 64 (1958), 87-89.

[4] J. A. Cuenca Mira, R. De Los Santos Villodres, A. Kaidi, and A. Rochdi, Real quadratic flexible division algebras, Linear Algebra Appl. 290 (1999), no. 1-3, $1-22$.

[5] J. A. Cuenca Mira and Á. Rodríguez-Palacios, Absolute values on $H^{*}$-algebras, Comm. Algebra 23 (1995), no. 5, 1709-1740.

[6] M. L. El-Mallah, Absolute valued algebras with an involution, Arch. Math. (Basel) 51 (1988), no. 1, 39-49.

[7] M. A. Kervaire, Non-parallelizability of the $n$-sphere for $n>7$, Proc. Natl. Acad. Sci. USA 44 (1958), 280-283.

[8] J. M. Osborn, Quadratic division algebras, Trans. Amer. Math. Soc. 105 (1962), 202-221.

[9] M. Ramírez Álvarez, On four-dimensional absolute-valued algebras, Proceedings of the International Conference on Jordan Structures (Málaga, 1997), Univ. Málaga, Málaga, 1999, pp. 169-173.

[10] A. Rochdi, Étude des algèbres réelles de Jordan non commutatives, de division, de dimension 8, dont l'algèbre de Lie des dérivations n'est pas triviale [Study of noncommutative Jordan real division algebras of dimension 8 whose Lie derivation algebra is nontrivial], J. Algebra 178 (1995), no. 3, 843-871 (French).

[11] Á. Rodríguez-Palacios, One-sided division absolute valued algebras, Publ. Mat. 36 (1992), no. 2B, 925-954.

[12] K. Urbanik and F. B. Wright, Absolute-valued algebras, Proc. Amer. Math. Soc. 11 (1960), 861-866.

A. Rochdi: Département de Mathématiques et Informatique, Faculté des Sciences, Université Hassan II, BP 7955, Casablanca, Morocco

E-mail address: abde11ati froc@hotmai 1 .com 


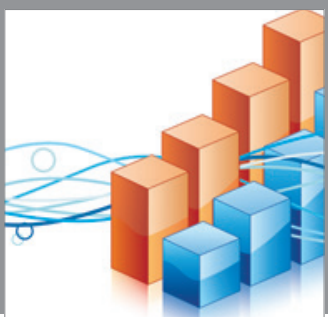

Advances in

Operations Research

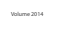

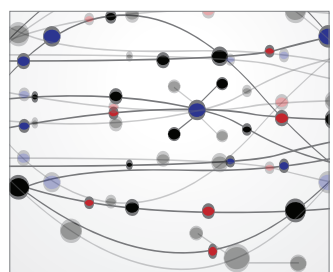

\section{The Scientific} World Journal
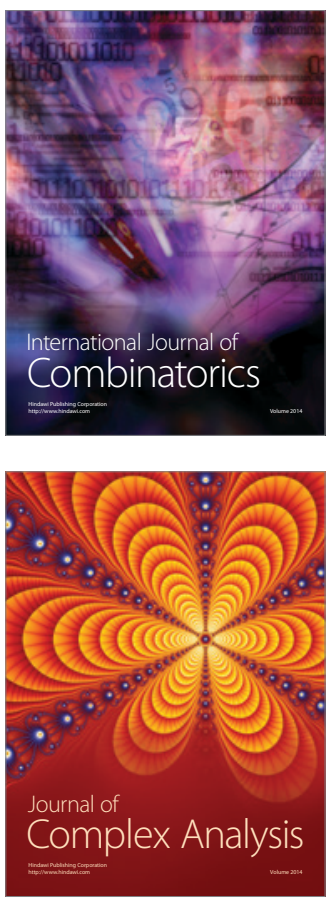

International Journal of

Mathematics and

Mathematical

Sciences
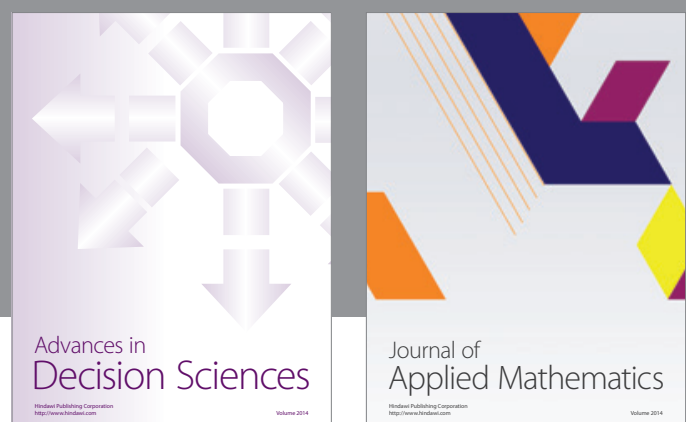

Journal of

Applied Mathematics
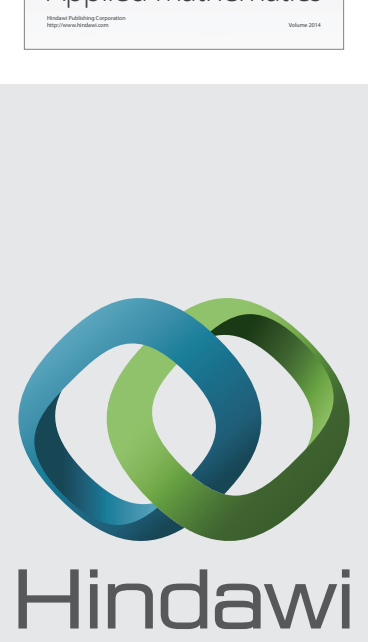

Submit your manuscripts at http://www.hindawi.com
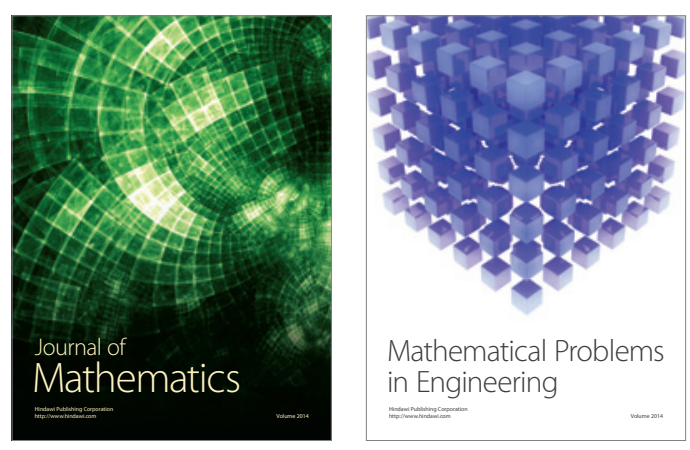

Mathematical Problems in Engineering
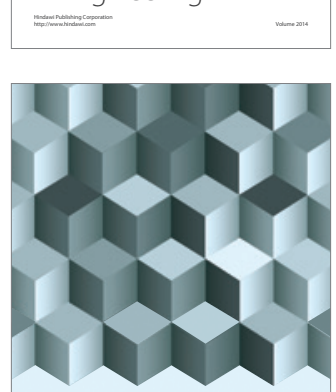

Journal of

Function Spaces
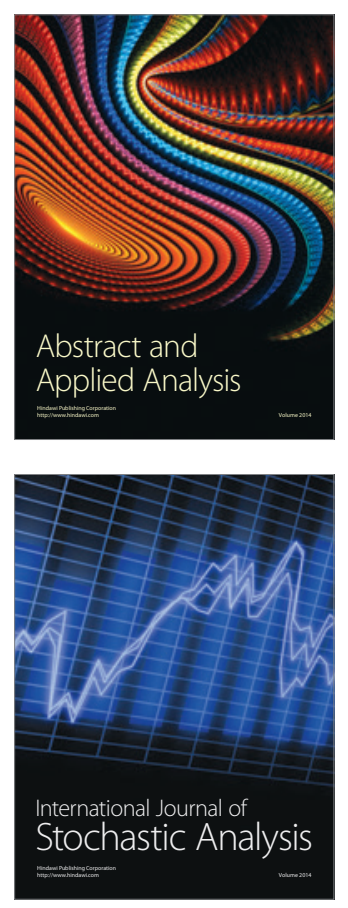

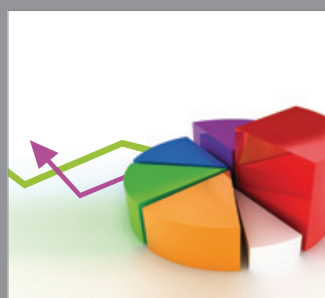

ournal of

Probability and Statistics

Promensencen
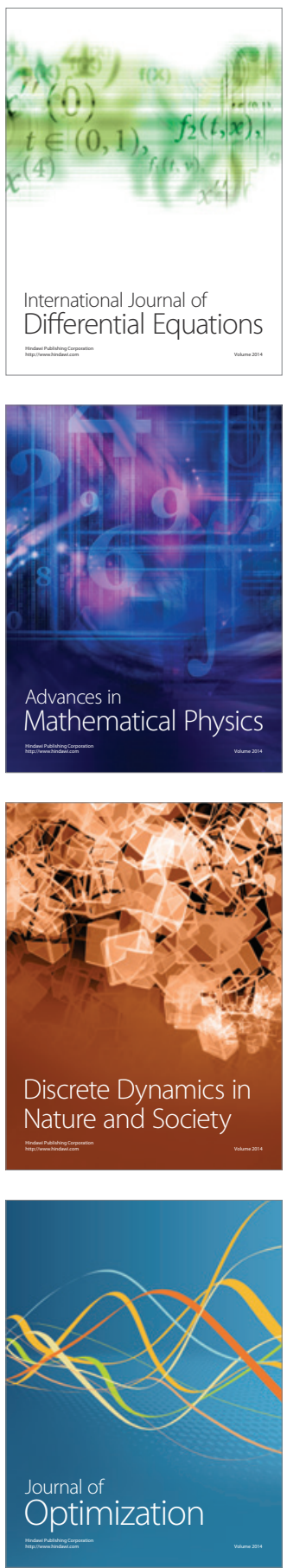